\title{
ENTORNO AVANZADO DE CO-SIMULACIÓN PARA MANIOBRAS COOPERATIVAS ENTRE VEHÍCULOS
}

\author{
Antonio Artuñedo, Jorge Godoy, Rodolfo Haber \\ Centro de Automática y Robótica (CSIC-UPM), Ctra. M300 Campo Real, Km 0,200, Arganda del Rey \\ \{antonio.artunedo, jorge.godoy, rodolfo.haber\}@car.upm-csic.es
}

\begin{abstract}
Resumen
El objetivo de este trabajo es el desarrollo de funcionalidades avanzadas sobre un simulador de maniobras cooperativas entre vehículos autónomos. Se trata de una plataforma de simulación genérica en la que el usuario puede definir el escenario deseado mediante una interfaz gráfica. Esta interfaz facilita la inserción de controladores y modelos para diferentes partes de los vehículos y de algunos elementos de la infraestructura, a través de una librería de bloques de Simulink. Además, permite las comunicaciones entre vehículos (V2V) y entre vehículos e infraestructura (V2I). El simulador posibilita la representación $3 D$ de la simulación y también la monitorización de ciertas variables en tiempo de simulación. La mayor ventaja del entorno propuesto es el uso de simulación hibrida combinando vehículos reales y virtuales en el mismo escenario de prueba, sin la necesidad de vehículos y equipos reales caros. Otra funcionalidad implementada es la recreación de una prueba experimental a partir de un conjunto de datos proveniente de los sensores de los vehículos. El simulador ha sido desarrollado bajo el entorno de programación Matlab/Simulink, sacando el máximo partido de la herramienta Simulink 3D Animation.
\end{abstract}

Palabras Clave: Co-simulación, Conducción autónoma, Simulación, Sistemas Inteligentes de Transporte, Tiempo real.

\section{INTRODUCCIÓN}

En últimas décadas, los avances en las nuevas tecnologías de la comunicación y en la capacidad de procesamiento de los ordenadores han dado lugar a nuevos desafíos para aplicaciones de sistemas inteligentes de transporte (ITS). Estos nuevos retos implican el desarrollo de nuevos sistemas para mejorar diferentes aspectos tales como la eficiencia, la fiabilidad, la seguridad y la seguridad en los ITS. Los ITS comprenden un conjunto de soluciones tecnológicas para las telecomunicaciones y las tecnologías de la información, destinadas a mejorar el funcionamiento y la seguridad del transporte. Una de las principales ramas del desarrollo de este conjunto de soluciones telemáticas está orientada principalmente al transporte por carretera. Dentro de la rama de transporte por carretera, los ITS hacen uso de comunicaciones vehículo-vehículo (V2V) y vehículo-infraestructura (V2I), así como de sistemas de percepción autónomos basados en sensores, como LIDAR, RADAR o cámaras para visión por computador. En general, los diferentes tipos de ITS se basan en las tecnologías de comunicación de radio y el uso de tecnologías especializadas.

El número de aplicaciones reportadas en el campo de los ITS no solo está creciendo rápidamente en la literatura, sino también en soluciones comerciales disponibles. Alguna de estas aplicaciones incluyen el transporte urbano, los sistemas adaptativos de control de tráfico (ATCS) [4, 8, 13], sistemas avanzados de información al viajero (ATIS) y aplicaciones para carga y logística [4] entre otros. Hoy en día, los sistemas de seguridad activa se centran principalmente en la forma de perfeccionar y mejorar los sistemas avanzados de ayuda al conductor (ADAS), que de hecho es una prioridad en la agenda de investigación y desarrollo para el sector académico e industrial.

En el ámbito de la investigación en el transporte y los ITS, la simulación y las metodologías de prototipado rápido tienen un papel clave. En base a esto, una herramienta para la simulación de una amplia gama de aplicaciones de ITS se propone en este trabajo. Este es un marco para la simulación de maniobras de cooperación entre los vehículos. Su objetivo es hacer frente a las nuevas necesidades en su simulación como la simulación de ADAS, pruebas de controladores para los vehículos autónomos o sensores inteligentes; mantenimiento de un equilibrio entre los simuladores de la dinámica del vehículo y simuladores de tráfico microscópicos.

Actualmente hay herramientas que permiten la simulación de tráfico por carretera mientras que otras se enfocan en la simulación de la dinámica de un vehículo. La mayoría de estas herramientas están enfocadas en diferentes aspectos del tráfico. Por lo general, estas herramientas utilizan modelos de tráfico macroscópicos o microscópicos. Por un lado, 
los modelos macroscópicos se centran en las relaciones globales de los flujos de tráfico, tales como la velocidad del vehículo, el flujo de tráfico y la densidad del tráfico. Algunos ejemplos de los simuladores macroscópicas son AIMSUN [1] o Visum [12]. Por otro lado, los modelos microscópicos consideran los aspectos dinámicos de cada vehículo de forma individual. Algunos de los simuladores microscópicos más conocidos son SUMO [9] y PTV VisSim [14]. Además otras herramientas de simulación se centran en la dinámica del vehículo. El comportamiento dinámico de los vehículos puede analizarse de diferentes maneras, que van desde un sistema de resorte-masa simple con tres grados de libertad, hasta un alto grado de complejidad. Uno de los más conocidos de este tipo de simulador son CarSim [2], y veDyna [6]. Sin embargo, es difícil encontrar una plataforma de simulación intermedio que considera el comportamiento del tráfico microscópico, sin olvidar la dinámica del vehículo.

Nuestra propuesta acorta la distancia entre los simuladores de tráfico y los simuladores de dinámica del vehículo permitiendo a investigadores $\mathrm{y}$ desarrolladores realizar pruebas de equipos embarcados en el vehículo, tales como sensores, actuadores o controladores, y maniobras cooperativas de transporte en escenarios urbanos realistas.

\section{DESCRIPCIÓN DEL ENTORNO DE SIMULACIÓN}

El entorno de simulación propuesto en este trabajo se enfoca en las maniobras cooperativas entre vehículos. Se trata de una plataforma de simulación genérica en la que el usuario puede definir el escenario deseado mediante una interfaz gráfica. Esta interfaz facilita la inserción de controladores y modelos para diferentes partes de los vehículos y de algunos elementos de la infraestructura, a través de una librería de bloques de Simulink. Además, permite las comunicaciones entre vehículos (V2V) y entre vehículos e infraestructura (V2I).

El simulador posibilita la representación 3D de la simulación y también la monitorización de ciertas variables en tiempo de simulación. La mayor ventaja del entorno propuesto es el uso de simulación hibrida combinando vehículos reales y virtuales en el mismo escenario de prueba, sin la necesidad de vehículos y equipos reales caros. Otra funcionalidad implementada es la recreación de una prueba experimental a partir de un conjunto de datos proveniente de los sensores de los vehículos. El marco de simulación ha sido implementado en Matlab/Simulink.
El marco está estructurado en diferentes componentes como se muestra en la figura 1. Esta figura muestra un diagrama funcional de la estructura. Cada una de estas partes se describe a continuación.

La interfaz de usuario principal simplifica la definición del escenario, permitiendo al usuario personalizar los parámetros de simulación en un ambiente amigable (ver figura 2). Las características más interesantes de esta GUI son la definición de la configuración de los vehículos, los enlaces de comunicaciones, infraestructura y el entorno 3D. En cuanto a los vehículos, controladores (longitudinal y lateral) y modelos para los diferentes componentes del vehículo, así como algunos elementos de la infraestructura son seleccionados a través de una biblioteca de bloques. Esta biblioteca se estructura como se muestra en la figura 3. Además, el usuario puede añadir nuevos bloques y modificar los existentes individualmente. La biblioteca proporciona una fácil integración de nuevos modelos para cualquier sección de la biblioteca (dinámica del vehículo, sensores, actuadores, etc.).

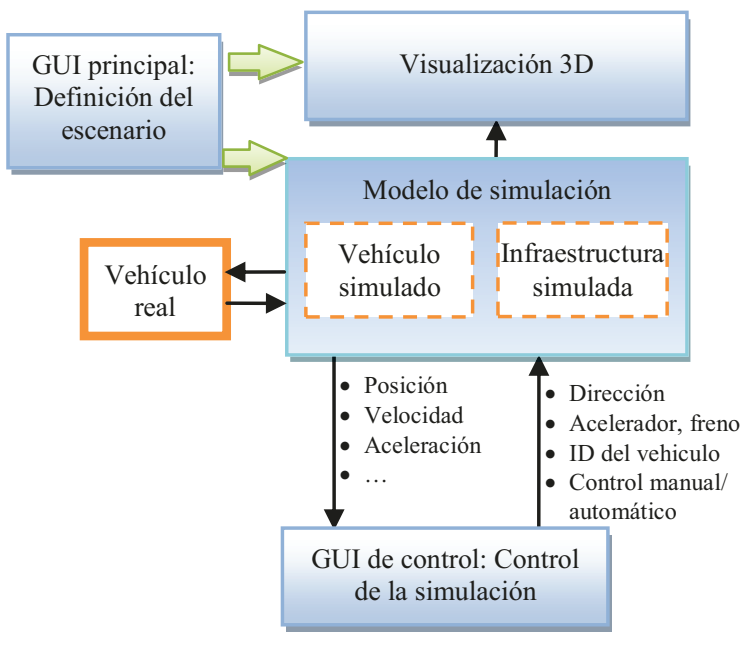

Figura 1: Diagrama general del marco de simulación

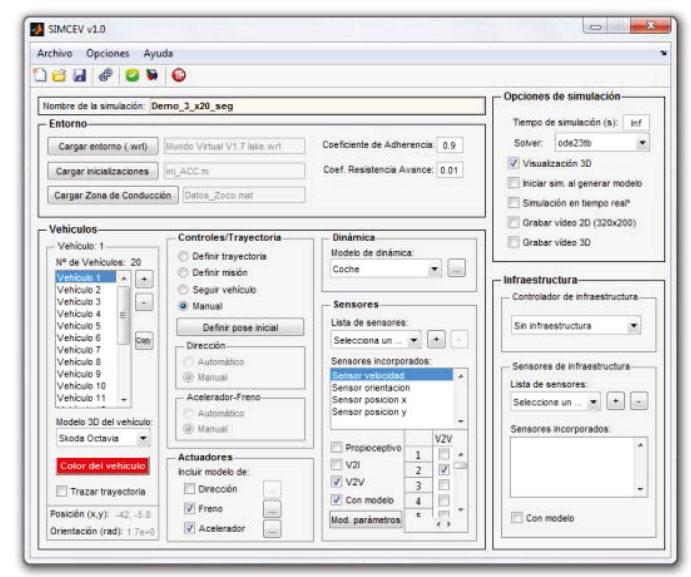

Figura 2: GUI principal 


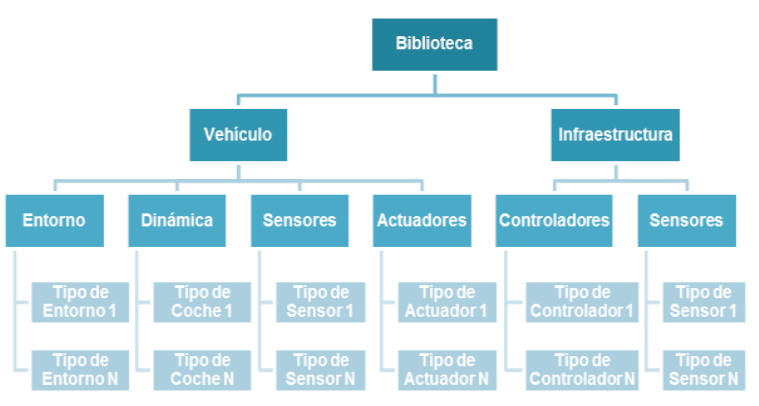

Figura 3: Esquema de la biblioteca de modelos

El simulador fue diseñado para que cada vehículo tenga un objetivo en la simulación: (i) completar una misión, (ii) seguir a otro vehículo, (iii) seguir un camino definido o (iv) de conducción manual. El objetivo seleccionado establece los requisitos para la definición del vehículo en el escenario de simulación. En el primer caso, completar una misión significa que el vehículo es capaz de ir desde el punto inicial hasta el punto final automáticamente, por lo que el usuario debe incluir controladores de conducción autónoma y especificar posición y orientación del vehículo tanto en el punto de partida como en el punto final. La segunda opción es adecuada para la simulación de sistemas ACC (Adaptive Cruise Control) o sistemas CACC (Cooperativa Adaptive Cruise Control). En este caso el objetivo del vehículo es seguir a otro vehículo por lo que se requieren controladores de conducción autónoma, el punto y orientación iniciales, y la identificación del vehículo líder.

En la simulación de tráfico, los vehículos por lo general siguen una trayectoria predefinida. Para estos casos, se debe utilizar la tercera opción. Se requiere que el usuario introduzca la ruta a través de la interfaz gráfica de usuario. Por último, la conducción manual permite al usuario controlar manualmente un vehículo simulado usando un mando o teclado. La definición de los vehículos a través de estas opciones proporciona una forma general para diseñar un escenario de simulación que contiene vehículos con diferentes objetivos y también vehículos conducidos manualmente. Como se describe en la sección 2.3, la interacción en real-virtuales entre vehículos proporciona nuevas características y amplía las capacidades del entorno de simulación.

Una vez que el escenario de simulación se ha definido, la herramienta genera automáticamente el modelo Simulink para la simulación y el entorno 3D. También se genera una segunda interfaz gráfica de usuario para el seguimiento de la evolución de las variables seleccionadas y el control manual de los vehículos en tiempo de ejecución.

Una representación general del flujo de trabajo con esta herramienta se representa en la figura 4.

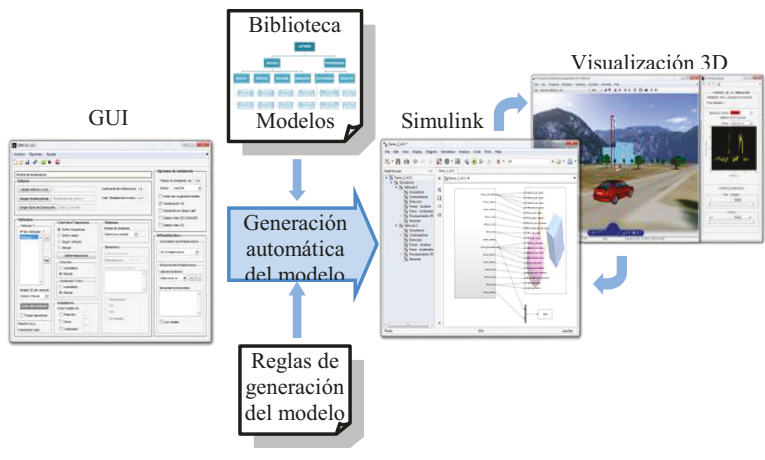

Figura 4: Flujo de trabajo

\subsection{ESTRUCTURA DEL MODELO}

Con el objetivo de facilitar la compresión del modelo al usuario y la interacción con el entorno de simulación, el modelo se genera siguiendo una estructura organizada por niveles tal y como se indica en la figura 5.

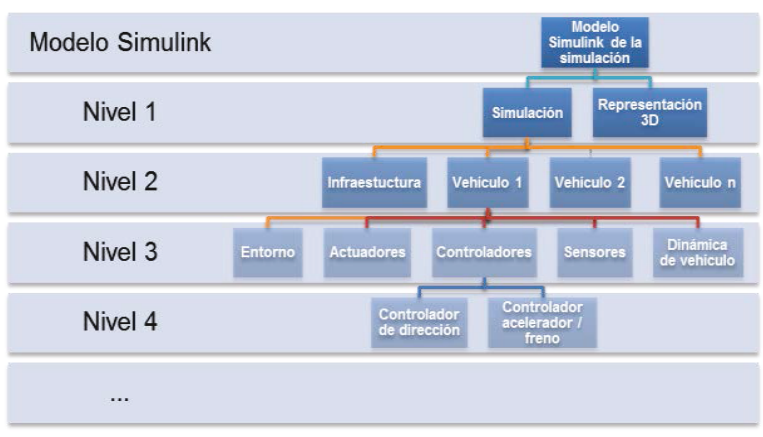

Figura 5: Estructura del modelo de simulación

En el primer nivel se encuentran únicamente dos bloques: Uno contiene el modelo de la simulación y el otro se encarga de la visualización 3D. En el segundo nivel se incluyen los elementos del escenario de simulación tales como vehículos y elementos de la infraestructura, cuyos componentes (sensores, actuadores, controladores, etc.) son especificados desde el nivel 3 en adelante.

\subsection{ENTORNO GRÁFICO}

Un componente importante del simulador es la visualización en $3 \mathrm{D}$ de la simulación. El entorno gráfico 3D se compone de un mapa y los vehículos en. El lenguaje de modelado 3D empleado para el diseño de todos los modelos en 3D que utiliza la herramienta es Virtual Reality Modeling Language (VRML). VRML es un lenguaje normalizado orientado a la visualización de objetos o escenas interactivas tridimensionales. VRML permite la visualización de una escena $3 \mathrm{D}$ compuesta de objetos 
de prototipos basados en formas geométricas básicas o estructuras donde las propiedades específicas se detallan: los vértices, bordes de cada polígono en tres dimensiones y su color de la superficie [3].

Las características de visualización 3D permiten a los usuarios simular escenarios influenciados por diferentes condiciones de conducción tales como baja visibilidad causada por las condiciones climáticas adversas, como la niebla, o diferentes situaciones de iluminación como de día o de noche. Esto permite la simulación de sensores que se utilizan comúnmente en el ámbito los vehículos autónomos, por ejemplo, sensores de procesamiento de imágenes para la detección de carriles u obstáculos, así como sensores de infraestructura para la gestión del tráfico y de vigilancia. En la figura 6 se puede ver un ejemplo de visualización 3D.

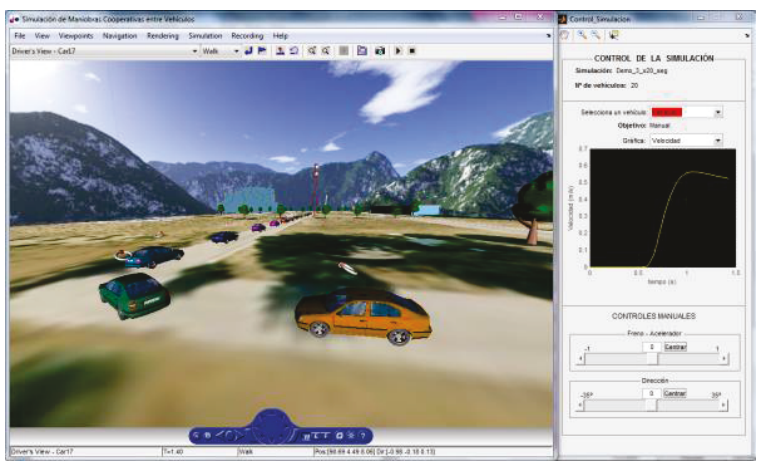

Figura 6: Visualización 3D e interfaz de control de la simulación

\subsection{TIEMPO REAL Y COMUNICACIONES EN CO-SIMULACIÓN}

Las aplicaciones distribuidas en entornos de sistemas de control requieren una ajustada sincronización para garantizar la entrega de mensajes de control dentro de los tiempos de ciclo de mensajes requeridos. Los aspectos temporales juegan un papel clave en las comunicaciones y por lo general son las principales limitaciones para este tipo de aplicaciones. En el ámbito de las aplicaciones de control para la conducción, los requisitos de tiempo están en el orden de milisegundos [5]. La limitación de tiempo no es problemática en este caso dado que solamente se comparte información entre vehículos reales y simulados y las acciones de control no se envían desde un PC (donde se ejecuta la simulación) a los vehículos.

Para la implementación de la plataforma de cosimulación se empleó el esquema presentado en la figura 7. El sistema se compone de un PC host (o anfitrión) encargado mostrar de la visualización 3D de la co-simulación así como la evolución de las variables seleccionadas de los vehículos (velocidad, aceleración, etc.), un PC target encargado de la simulación en tiempo real, un sistema de comunicaciones y los vehículos reales.

\section{EVALUACIÓN}

Para analizar el resultado obtenido con la plataforma de simulación y validar el modelo del vehículo utilizado, varios experimentos fueron llevados a cabo. En estos experimentos se usó un coche real y dos simulados. El coche real es un Citroën C3 adaptado para la conducción autónoma, incluyendo varios sensores como GPS, cámaras, unidad de medición inercial (IMU); y actuadores en el acelerador, freno y volante. El vehículo también cuenta con un ordenador embarcado que está a cargo del control del vehículo y un sistema de comunicaciones con el protocolo IEEE 802.11p para las comunicaciones V2V y V2I [10].

El modelo de vehículo empleado para los dos vehículos simulados incluye modelos para el motor, caja de cambios automática y dinámica del vehículo, donde se consideran fuerzas de fricción y aerodinámica. El modelo se ajustó para imitar el comportamiento del vehículo real usado en las pruebas.

El escenario de pruebas consistió en una flota de vehículos realizando un seguimiento de la trayectoria del líder mientras que circulan en ACC (Adaptive

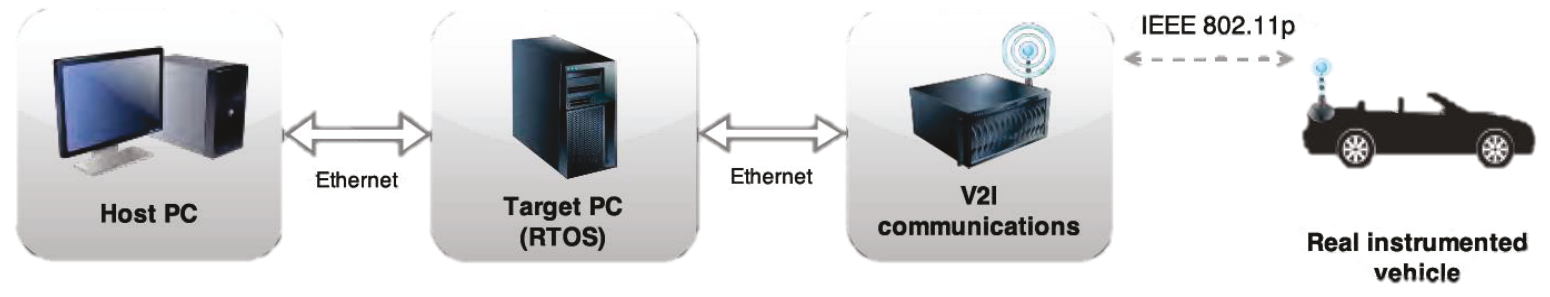

Figura 7: Componentes de la implementación en tiempo real 
Cruise Control). De tal manera que los objetivos individuales de cada vehículo fueron los siguientes: (i) el vehículo líder (simulado) seguía una trayectoria predefinida. (ii) el segundo (real), iba siguiendo al primer vehículo. Por último, (iii) el tercer coche iba siguiendo al segundo. Todos los vehículos usaron los mismos controladores laterales y longitudinales usados en el experimento de $100 \mathrm{~km}$ realizado previamente con dos coches reales [7].

La figura 8 muestra las trayectorias seguidas por los tres vehículos a lo largo de la prueba. Los resultados del experimento demostraron que es posible la cooperación entre vehículos simulados y vehículos reales.

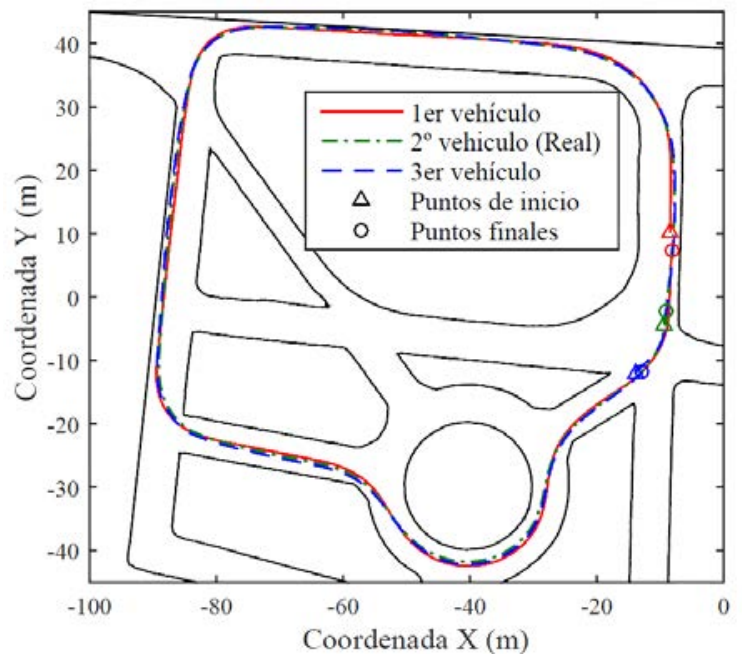

Figura 8: Trayectorias de los vehículos en la prueba de co-simulación

\section{CONCLUSIONES}

Esta comunicación propone un marco de simulación para el desarrollo de aplicaciones en el campo de la conducción autónoma. La herramienta propuesta tiene una estructura modular que permite al usuario implementar varios modelos y componentes en diferentes niveles del entorno de simulación. Además, simulador permite introducir conexiones entre vehículos y sistema de infraestructura que permite la comunicación entre ellos. También se permite la visualización 3D de la simulación.

Los resultados muestran que la herramienta propuesta hace posible la interacción en tiempo real entre vehículos reales y virtuales permitiéndoles cooperar.

Los trabajos futuros se centrarán en la mejora de los modelos usados en el simulador así como la inclusión de nuevos modelos. Por otra parte, la funcionalidad que hace posible la cooperación en tiempo real entre vehículos reales y virtuales permite usar la herramienta como una capa de abstracción de hardware para futuros desarrollos y pruebas de sistemas ciber-físicos en el campo de los ITS.

\section{Agradecimientos}

Este trabajo contó con el apoyo de los proyectos RTC-2015-3942-4 "Family of High-Performance Compact Cards for Automotive Applications" (TCAP-Auto) y DPI2014-53525-C3-3-R: "Extension of Cognitive Capabilities to the navigation of robots in non-structured Environments" (NAVEGASE), ambos financiados por el Ministerio de Economía y Competitividad (MINECO) y financiación FEDER.

\section{Referencias}

[1] AIMSUN, N., "AIMSUN Micro Version 5.1, TSS-Transport Simulation Systems," ed, 2006.

[2] Arbor, A., "Carsim Reference Manual, Ver. 6.03," ed: Toyota company, Japan: Mechanical Simulation Corporation, 2005.

[3] Consortium, W. D. (2004). International Standard ISO/IEC 14772-1:1997 and ISO/IEC 14772-2:2004. Available: http://www.web3d.org/content/vrm197functional-specification-and-vrm197-externalauthoring-interface-eai

[4] de Oliveira, L. B. y Camponogara, E., "Multiagent model predictive control of signaling split in urban traffic networks", (2010) Transportation Research Part C: Emerging Technologies, vol. 18, pp. 120-139.

[5] Doyle, P., "Introduction to Real-Time Ethernet I", (2004) The Extension - A Technical Supplement to Control Network, vol. 5,

[6] Dynaware, T., "veDyna 3.10 User Manual," ed: München, 2007.

[7] Godoy, J., Pérez, J., Onieva, E., Villagrá, J., Milanés, V., y Haber, R., "A driverless vehicle demonstration on motorways and in urban environments", (2015) Transport, pp. 1-11.

[8] Hamilton, A., Waterson, B., Cherrett, T., Robinson, A., y Snell, I., "The evolution of urban traffic control: changing policy and technology", (2013) Transportation Planning and Technology, vol. 36, pp. 24-43.

[9] Krajzewicz, D., Hertkorn, G., Rössel, C., y Wagner, P., "Sumo (simulation of urban mobility)," in Proc. of the 4th Middle East Symposium on Simulation and Modelling, 2002, pp. 183-187.

[10] Milanés, V., Naranjo, J. E., González, C., Alonso, J., y de Pedro, T., "Autonomous vehicle 
based in cooperative GPS and inertial systems", (2008) Robotica, vol. 26, pp. 627-633.

[11] P, J., \#233, rez, D, J., \#237, az, Garbajosa, J., Agust, Yag, n., \#252, Gonzalez, E., y LopezPerea, M., "Large-scale smart grids as system of systems," presented at the Proceedings of the First International Workshop on Software Engineering for Systems-of-Systems, Montpellier, France, 2013.

[12] PTV, A., "VISUM 11 User Manual", (2009) Karlsruhe, Germany,

[13] Robertson, D. I. y Bretherton, R. D., "Optimizing networks of traffic signals in real time-the SCOOT method", (1991) Vehicular Technology, IEEE Transactions on, vol. 40, pp. 11-15.

[14] VISSIM, P., "5.10 User Manual", (2008) PTV Vision, July, vol. 18, 